\title{
Biochemical Induction of Fruiting Bodies in Schizophyllum commune: a Bioassay and its Application
}

\author{
By S. RUSMIN AND T. J. LEONARD* \\ School of Biological Sciences, University of Kentucky, \\ Lexington, Kentucky 40506, U.S.A.
}

(Received 30 December 1974; revised 17 March 1975)

\section{SUMMARY}

A bioassay was developed for the study of the biochemical induction of homokaryotic fruiting in Schizophyllum commune. A fruiting-inducing substance (FIS) was incorporated into a small cylindrical agar block which was used to induce the test colony grown on cellophane membrane. The location, time of fruiting and number of fruiting bodies were predictable. This assay system was employed to study the process of fruiting induction. Although colonies up to 9 days old could be induced to fruit, the induced response was restricted in these colonies to hyphae less than $24 \mathrm{~h}$ old at the time of FIS exposure. Light was necessary during a period following FIS application for fruiting to occur. High temperature $\left(30^{\circ} \mathrm{C}\right)$ was found to inhibit FIS-induced fruiting but did not prevent FIS uptake by the colony.

\section{INTRODUCTION}

Schizophyllum commune is a heterothallic basidiomycete that forms small fruiting bodies on rotting wood. It is easily cultured on simple media and completes all the phases of its life cycle within 7 days. The formation of the fruiting bodies generally occurs as a result of a compatible mating between homokaryotic mycelia that are heterozygous for the mating loci. The resulting heterokaryotic cells produce a dikaryotic colony that develops dikaryotic fruiting bodies in which nuclear fusions, meiosis and sporulation occur.

Although fruiting bodies are generally formed by dikaryotic hyphae, some strains of $S$. commune produce homokaryotic fruiting structures that are indistinguishable macroscopically from those produced on dikaryotic colonies. Homokaryotic fruiting bodies are, however, composed of hyphae that are uninucleate, lack clamp connexions and produce fewer spores than their dikaryotic counterparts (Raper, 1966). The nature of the nuclear cycle in homokaryotic basidia during sporulation has not as yet been elucidated.

Physiological mechanisms regulating the formation of reproductive structures in basidiomycetes with large fruiting bodies are largely unknown. There is a vast literature on the effects of environmental factors such as light, temperature and nutrition on mushroom formation (see Taber, 1966). Although biochemical regulation of fruiting has been suggested (Urayama, 1956; Bevan \& Kemp, 1958; Eger, I965; Leonard \& Dick, I968; Uno \& Ishikawa, 1973), no hormonal systems comparable with those in Achlya and Mucor have been demonstrated.

Diffusible factors of low molecular weight, which induced fruiting in otherwise vegetative homokaryotic strains of $S$. commune, were isolated by Leonard \& Dick (1968) from the mycelium of Cladosporium cladosporoides (Fungi Imperfecti) and from the fruiting bodies

\footnotetext{
* Present address: Department of Botany, University of Wisconsin, Madison, Wisconsin 53706, U.S.A.
} 


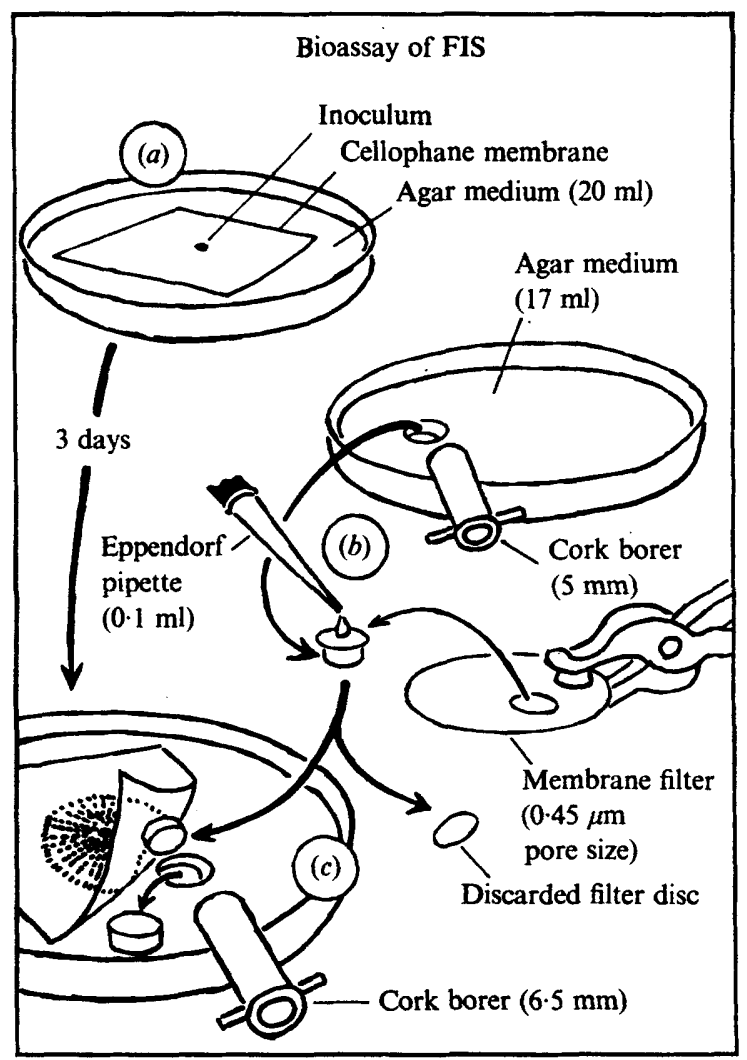

Fig. I. Preparation of the test colony and agar cylinder, and placing the agar cylinder beneath the test colony. Quantities as low as $0.01 \mathrm{ml}$ could be bioassayed. Sterilization of the inducing solution took place as it passed through the bacterial retaining filter before diffusing into the agar cylinder.

of $S$. commune and Agaricus bisporus (the cultivated mushroom). None of these factors has been purified, but for convenience they are referred to as a single fruiting-inducing substance, FIS. The purification of these factors and the study of FIS induction have been hampered by the lack of a sensitive bioassay to detect FIS activity. The existing assay (Leonard \& Dick, 1968) required relatively large amounts of FIS-containing solutions, regardless of FIS concentration, to elicit homokaryotic fruiting in the test colony.

We report a sensitive, quantitative microtechnique to assay FIS activity, and the effects of certain physiological and environmental factors on biochemically-induced homokaryotic fruiting.

\section{METHODS}

Organism and medium. Schizophyllum commune strain LI5 was used throughout these experiments. This homokaryon was isolated from a dikaryon fruiting body; the dikaryon was composed of two highly isogenized homokaryons (699 and E908) with the exception of mating type. These strains were obtained from the late Professor J. R. Raper of Harvard University. Strain L15 has the ability to produce haploid fruiting bodies under certain conditions (Leonard \& Dick, 1973). The medium contained (g/l distilled water): glucose, IO; DL-asparagine, I.0; $\mathrm{K}_{2} \mathrm{HPO}_{4}$, I.0; $\mathrm{KH}_{2} \mathrm{PO}_{4}, 0.46 ; \mathrm{MgSO}_{4} \cdot 7 \mathrm{H}_{2} \mathrm{O}, 0.5$; thiamine hydro- 
chloride, $1 \cdot 2 \times 10^{-4}$; agar, 20.0. The medium was sterilized by autoclaving for $15 \mathrm{~min}$ at $12{ }^{\circ} \mathrm{C}$; the glucose was sterilized separately to minimize caramelization.

Assay method. Figure I illustrates the assay method. All operations were conducted aseptically. In Fig. I $(a)$, the test colony was prepared by placing a piece of $5 \times 5 \mathrm{~cm}$ cellophane membrane (Du Pont CNC 2495 No. 7350, weight 150 poundals, Du Pont Laboratories, Chicago, Illinois, U.S.A.) on $20 \mathrm{ml}$ medium in a $10 \mathrm{~cm}$ Petri plate and inoculating the plate with an agar cube (approx. $1 \mathrm{~mm}^{3}$ ) cut from the margin of an actively growing 5-day-old colony. Before use the membrane was treated by autoclaving at $12 \mathrm{I}{ }^{\circ} \mathrm{C}$ for $25 \mathrm{~min}$ in $5 \%$ $\mathrm{Na}_{2} \mathrm{CO}_{3}$ solution, and rinsed overnight with tap water and for an hour with distilled water. The cultures were incubated at room temperature in the dark for 3 days.

In Fig. I (b), a $5 \mathrm{~mm}$ diameter agar cylinder was cut from $17 \mathrm{ml}$ medium in a $10 \mathrm{~cm}$ Petri plate. A paper puncher was used to cut a $6.5 \mathrm{~mm}$ diameter disc of bacterial retaining membrane filter (G-S type, Millipore). The disc was placed on top of the agar cylinder, and $0.0 \mathrm{Iml}$ of the solution to be assayed was delivered on to the centre of the disc with an Eppendorf pipette (Brinkman Instrument, Inc., Westbury, New York, U.S.A.). The solution was imbibed into the agar cylinder through the filter disc within $30 \mathrm{~min}$.

In Fig. I $(c)$, the position of the hyphal front facing one corner of the cellophane membrane was marked on the bottom of the culture plate. The membrane in this same corner was lifted and a $6.5 \mathrm{~mm}$ diameter well made directly above the marking. Before lowering the membrane, the agar cylinder was placed at the underside of the membrane. The position of the cylinder was adjusted until I to $2 \mathrm{~mm}$ of the colony margin was over the cylinder, so that when the membrane was lowered the cylinder was suspended inside the well without touching the surrounding medium.

The induced culture was placed upright under a bank of fluorescent lamps (60 to 80 $\mathrm{ft}$-candle) at room temperature. Cultures were examined in the area over the inducing cylinder for a fruiting reaction over a period of 5 days. The fruiting response was expressed in terms of the number of fruiting structures, stages I to V, as described by Leonard \& Dick (1968).

Preparation of the inducing extract. A standard solution was prepared from an extract of A. bisporus fruiting bodies as follows: Fresh mushrooms $(\mathrm{I} 00 \mathrm{~g})$ were cleaned with distilled water and sliced longitudinally into 3 to 4 pieces per fruiting body. The slices were autoclaved at $121{ }^{\circ} \mathrm{C}$ for $15 \mathrm{~min}$ and produced about $30 \mathrm{ml}$ yellow cell-free extract. Additional extract was pressed from the tissue residue through a piece of cheesecloth. The residue was reextracted twice with $2 \times 30 \mathrm{ml}$ glass-distilled water. The pooled extract was flash-evaporated under vacuum at $50^{\circ} \mathrm{C}$ to a small volume, which was then reconstituted with glassdistilled water to $10 \mathrm{ml}$.

Light experiments. Light treatment was given by placing the plates under a bank of coolwhite fluorescent lamps ( 60 to $80 \mathrm{ft}$-candle), with daylight excluded. The room temperature during treatment was $25 \pm 2{ }^{\circ} \mathrm{C}$. Dark treatment was given by placing cultures in a drawer in a dark room. Manipulation for the biochemical induction of fruiting was performed in dim red light from a $14 \mathrm{~W}$ cool-white fluorescent lamp covered with two layers of red cellophane and placed about I $\mathrm{m}$ away.

Temperature experiments. Temperature treatment was carried out on shelves in a Psychrotherm incubator (model G-27, New Brunswick Scientific Co., New Brunswick, New Jersey, U.S.A.) equipped with temperature control and a bank of fluorescent lamps which delivered 60 to $80 \mathrm{ft}$-candle at the level of the culture plates. 


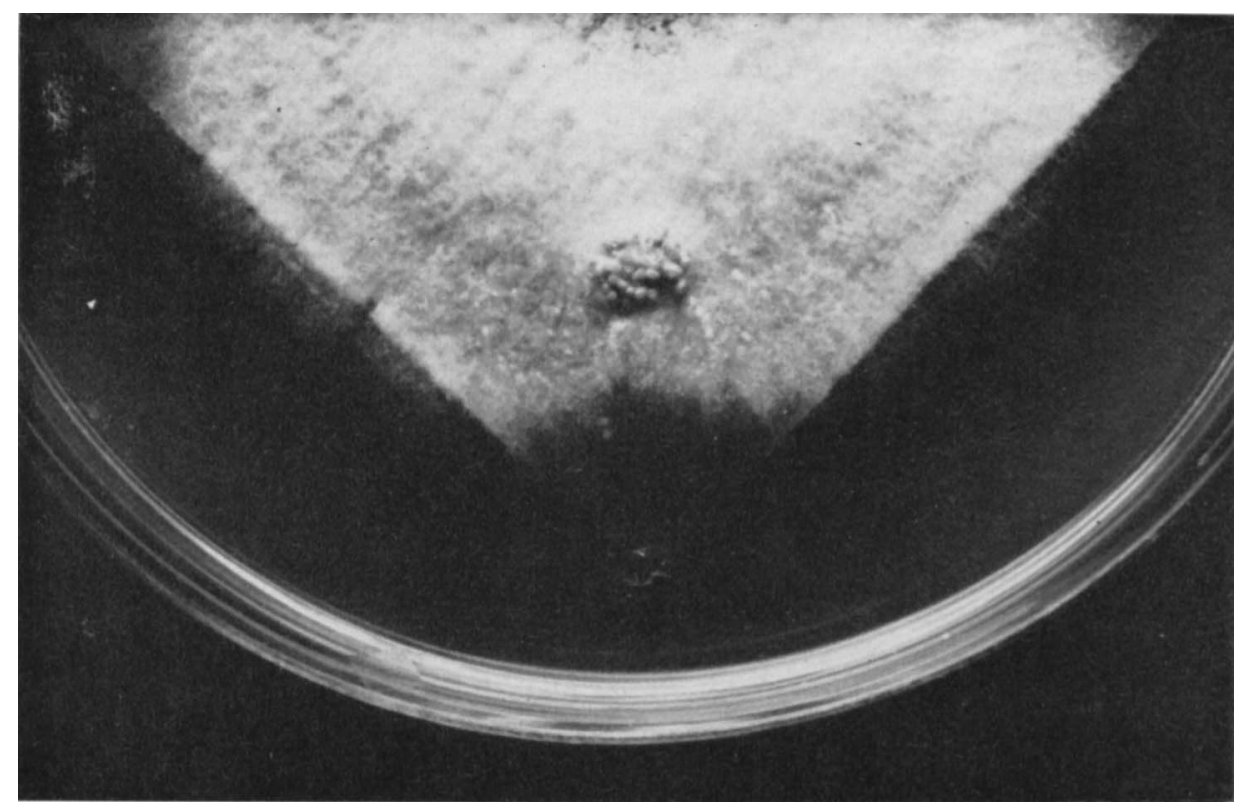

Fig. 2. The test colony on the 4th day after induction. Fruiting bodies of stages II and III developed in a restricted fruiting area. Approximately actual size.

\section{RESULTS}

The induced homokaryotic fruiting response

A typical induced fruiting reaction by the assay method is shown in Fig. 2. Fruiting bodies of stages II and III can be observed in a restricted area of the colony above the inducing cylinder. Similarly treated colonies which had not been exposed to FIS-containing solutions remained vegetative.

\section{Quantitative bioassay}

An FIS-containing extract was prepared and concentrated to $0.03 \mathrm{ml} / \mathrm{g}$ fresh mushrooms. This concentration was the highest attainable before the extract became too viscous to be delivered quantitatively with a pipette. Water dilutions of $1 / 3,1 / 10,1 / 30$ and $1 / 100$ the concentration of this extract were prepared. These preparations and the original extract were each bioassayed in 26 replicate induction plates. The results showed that a concentration of $1 / 100$ was too dilute to induce any fruiting response, whereas extracts of higher concentration induced increasing numbers of fruiting bodies with increasing concentration.

The spread in the number of fruiting bodies found on different induction plates at the same concentration was high, as indicated by the ranges (the difference between the highest and the lowest count), which were $6, \mathrm{II}, \mathrm{I} 7$ and $5 \mathrm{I}$ for concentrations of $\mathrm{I} / 30, \mathrm{I} / \mathrm{IO}, \mathrm{I} / 3$ and $\mathrm{I}$, respectively. A linear relationship between the concentration of inducing solution (dose) and the number of fruiting bodies (response) was best approached when logarithmic transformation was performed on data of both dose and response. Moreover, the transformation reduced the degree of data dispersion for higher doses to conform more with that of lower doses (Fig. 3).

Analysis of variance was performed on the logarithmically transformed data (Table $\mathrm{r}$ ). 


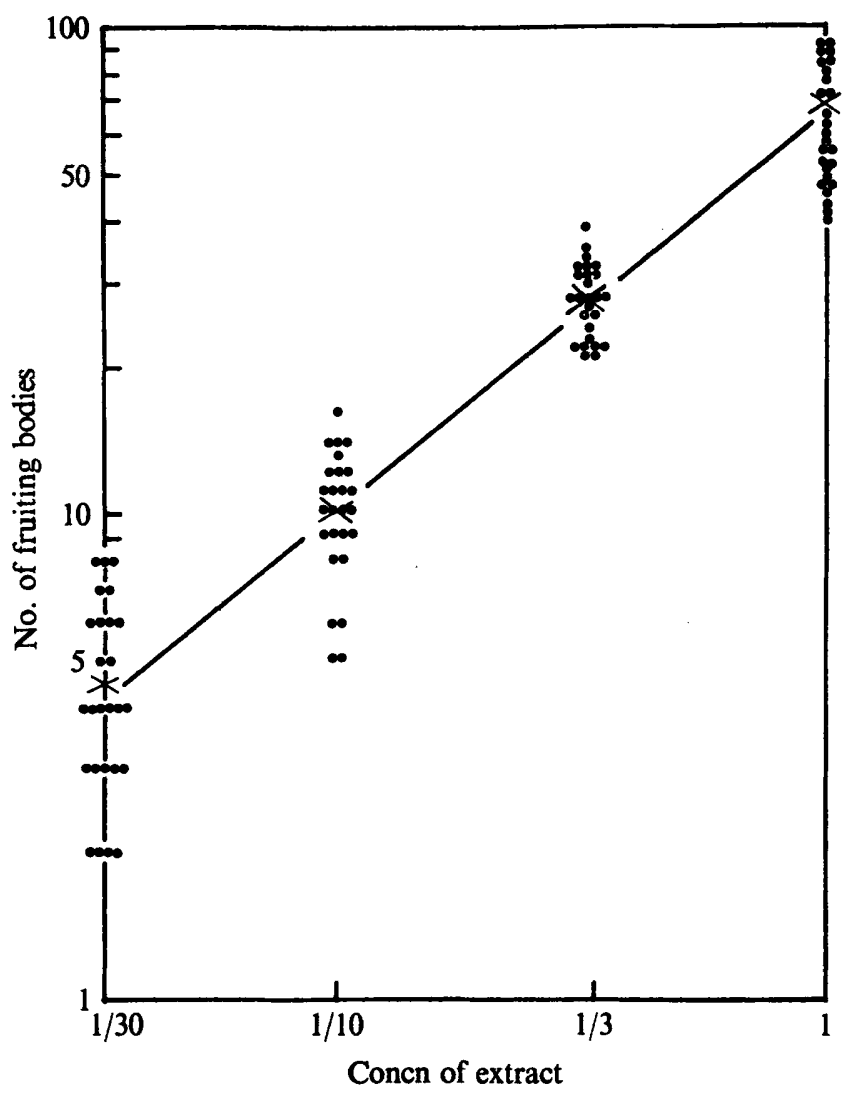

Fig. 3. The number of fruiting bodies plotted against extract concentration. Both axes are on a logarithmic scale. The straight line was calculated from the least mean squares. The number of fruiting bodies found, in each bioassay at the different concentrations, is represented by $\odot ; \times$ represents the mean number of fruiting bodies at a given concentration. The concentration of $I$ is equivalent to $0.03 \mathrm{ml}$ extract/g fresh mushrooms. The means of the number of fruiting bodies and their standard deviations, for the 26 test replicates at each concentration, are $4 \cdot 58 \pm 1 \cdot 96,10 \cdot 19 \pm$ $2 \cdot 84,27 \cdot 69 \pm 4 \cdot 60$, and $64.46 \pm 17 \cdot 59$, for concentrations of $1 / 30,1 / 10,1 / 3$, and 1 , respectively.

F-distribution tests showed that both regression and linearity of regression were highly significant. The equation for linear regression was calculated to be:

$$
y=0.61810+0.80128 x \text {, }
$$

where $y$ is the log number of fruiting bodies and $x$ the log extract concentration. The linear relationships between $\log$ dose and $\log$ response held true in repeated experiments.

The following series of experiments utilized the above assay method and a standard concentration of FIS to measure the effects of physiological and environmental factors on the induced homokaryotic fruiting response.

\section{Inducible region of a colony}

A fungal colony growing from a point inoculum at the centre of an agar medium consists of concentric regions of mycelia of different ages, the youngest at the edge of the colony, the oldest around the inoculum site; regions of different ages differ physiologically (Park \& 

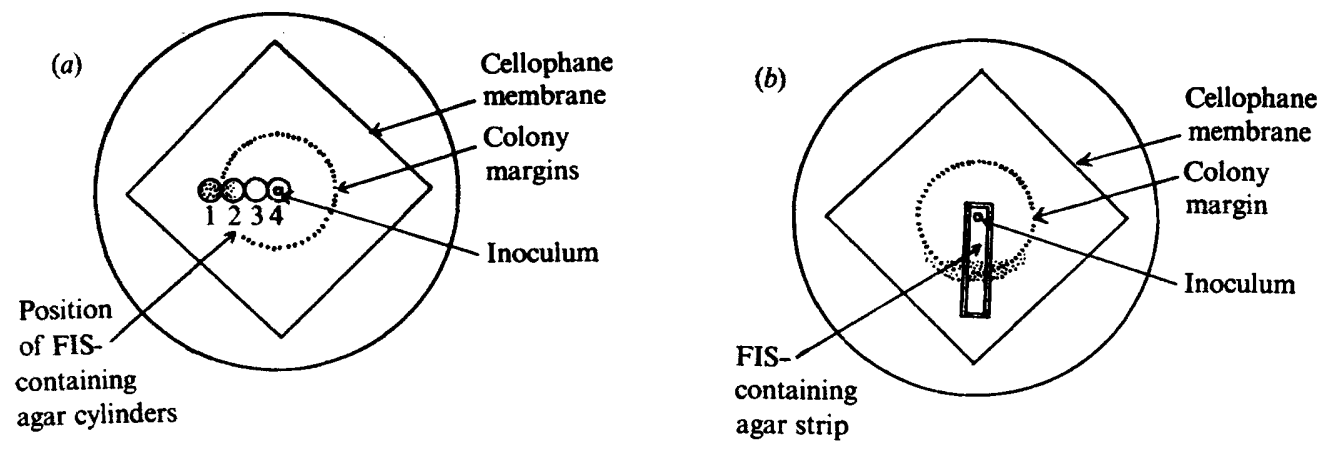

Fig. 4. Diagrams showing methods of inducing different regions of a colony with (a) FIS agar cylinders and $(b)$ an FIS agar strip. Fruiting bodies developed in the stippled areas.

Table I. Analysis of variance on logarithmically transformed data of dose (concentration of extract) and response (number of fruiting bodies)

$\begin{array}{lcccc}\text { Source } & \begin{array}{c}\text { Degrees } \\ \text { of freedom }\end{array} & \begin{array}{c}\text { Sum of } \\ \text { squares }\end{array} & \begin{array}{c}\text { Mean } \\ \text { squares }\end{array} & \text { F-distribution* } \\ \text { Between doses } & 3 & 20.5060 & 6.8535 & - \\ \quad \text { Linear regression } & \text { I } & 20.4936 & 20.4936 & 3305 \\ \text { Deviation from regression } & 2 & 0.0123 & 0.0062 & 0.3246 \\ \text { Within doses } & 100 & 1.9066 & 0.0191 & - \\ \text { Total } & \text { I03 } & 22.4126 & - & - \\ & \text { * Sokal \& Rohlf (1969). }\end{array}$

Robinson, 1966; Burnett, 1968). To determine the response of different regions to FIS induction, the following experiments were conducted.

FIS-containing agar cylinders were placed (Fig. $4 a$ ) at one of the following locations beneath 3-day-old membrane-grown colonies: (I) the edge of the colony, (2) the outermost $5 \mathrm{~cm}$ region of the colony, (3) the mid-point between the hyphal front and the inoculum, and (4) the inoculum. Only in the areas (I) and (2) did fruiting structures develop over the inducing cylinder. The mycelium in area (I) was in the process of growing over the cylinder and in area (2) was less than $24 \mathrm{~h}$ old at the time of induction.

Similarly, when an entire radius of the colony, from the inoculum point to the colony periphery, was simultaneously induced with a $4 \times 25 \mathrm{~mm}$ rectangular FIS-containing agar strip (Fig. $4 b$ ), fruiting response occurred in regions corresponding to areas (I) and (2) of Fig. 4(a). Although the inducing strip extended beyond the colony periphery, no fruiting structures were produced by the mycelium that colonized this area. However, hyphal areas lateral to the inducing strip (represented by the stippled area of Fig. $4 b$ ) were triggered to form fruiting bodies. This spreading of the fruiting agar was also observed when high concentrations of FIS were used with agar cylinders. That the age as well as the size of the colony could modify the induced fruiting reaction was suggested by the following observations. Colonies younger than 5 days old responded to FIS induction by producing a predictable number of fruiting structures, whereas colonies 5, 7 and 9 days old exhibited a weaker response or no response. 
Table 2. Fruiting induction by FIS cylinders in a second colony after various times of contact with a first colony

\begin{tabular}{|c|c|c|c|c|c|c|}
\hline \multirow{3}{*}{$\begin{array}{l}\text { Time of } \\
\text { contact } \\
\text { with the } \\
\text { first colony } \\
\text { (h) }\end{array}$} & \multicolumn{5}{|c|}{ All cultures were scored 8 days after inoculation. } & \\
\hline & \multicolumn{3}{|c|}{ First colony } & \multicolumn{3}{|c|}{ Second colony* } \\
\hline & Fruiting response $†$ & Mean & S.D. & Fruiting response $†$ & Mean & S.D. \\
\hline 6 & $0,0,0,0,0,0$ & 0.00 & 0.00 & $18,17,20,28,22,16$ & $20 \cdot 17$ & 4.40 \\
\hline 12 & $17,15,10,11,13,12$ & $13 \cdot 00$ & 2.6r & $15,8,11,9,8,10$ & $10 \cdot 17$ & $2 \cdot 64$ \\
\hline 18 & $22,10,19,19,20,14$ & $17 \cdot 33$ & $4: 46$ & $6,5,3,4,7,3$ & $4 \cdot 67$ & $r \cdot 63$ \\
\hline 24 & $18,25,21,12,20,20$ & $19 \cdot 33$ & $4 \cdot 27$ & $0,0,0,0,0,0$ & 0.00 & 0.00 \\
\hline 36 & $14,26,22,27,24,20$ & $22 \cdot 17$ & $4 \cdot 75$ & $0,0,0,0,0,0$ & 0.00 & 0.00 \\
\hline 48 & $27,16,20,24,21,18$ & $2 \mathrm{I} \cdot 00$ & 4.00 & $0,0,0,0,0,0$ & $0 . \infty$ & 0.00 \\
\hline
\end{tabular}

Duration of contact and depletion of FIS from inducing cylinders

To determine the depletion of FIS activity in the inducing cylinders (which may indicate the rate of FIS uptake by the test colony) two sets of cultures were prepared and one set was induced with FIS for different times. The inducing cylinders from this set were subsequently used to induce the second set of 3-day-old colonies, which were exposed to the inducing cylinders for 5 days and scored for a fruiting reaction (Table 2). In both sets of cultures the position of the inducing cylinder was as in area (I) of Fig. 4(a).

No differentiation was observed in cultures exposed to FIS for $6 \mathrm{~h}$. During this time, however, only $25 \%$ of the cylinder had been colonized. Complete overgrowth of the inducing cylinder did not occur until 15 to $20 \mathrm{~h}$ after the start of induction. Increased amount and duration of mycelial contact with the inducing cylinder enhanced the fruiting response. The maximum response was observed in colonies exposed to FIS for $36 \mathrm{~h}$.

The fruiting response in the second set of cultures demonstrated that $6 \mathrm{~h}$ contact with the first colonies did not appreciably decrease the FIS activity in the inducing cylinder. This suggested that diffusion of FIS by way of the cellophane membrane was small, if not negligible. Once the mycelium of the first colony completely colonized the cylinder, however, FIS activity in the cylinder was rapidly depleted, and $24 \mathrm{~h}$ after the start of induction the cylinder failed to elicit cell differentiation in the second colony. That suppression of fruiting in the second colony by inhibitors carried over from the first colony could be discounted, was shown as follows: after $24 \mathrm{~h}$ contact with the first colony, the inducing cylinder was 'recharged' with FIS activity and used to induce the second colony; no inhibition was detected.

\section{The effect of light on FIS induction}

Test cultures were treated with different combinations of light and dark during the periods before and after placing the FIS-containing agar cylinders. The results are shown in Table 3. Light exposure before contact with FIS had no apparent effect on the number or the stage of fruiting development attained. However, light was essential during the induction period, for cultures exposed to light during this period formed stage II and III structures whereas dark-grown cultures remained vegetative.

A set of 3-day-old dark-grown cultures was exposed to inducing cylinders and placed immediately in the dark. At various times thereafter, groups of six plates were exposed to 
Table 3. The effect of light and dark treatment on FIS-induced fruiting

Pre-
induction*
incubation*

(3 days) (5 days)

$\begin{array}{rrr}\text { Fruiting response } \dagger & \text { Mean } & \text { S.D. } \\ 29,38,35,27,32,33 & 32 \cdot 33 & 3.98 \\ 36,35,22,24,31,20 & 28 \cdot 00 & 6.90 \\ 0,0,0,0,0,0 & 0.00 & 0.00 \\ 0,0,0,0,0,0 & 0.00 & 0.00\end{array}$

* D, Dark; L, light.

$\dagger$ No. of stage II and III fruiting bodies/fruiting area of 6 replicate test plates at 8 days.

Table 4. Effect of different times of dark treatment on the FIS-induced fruiting response

All cultures were in darkness for the 3 days preceding induction.

\begin{tabular}{crrr} 
Period of darkness* (h) & \multicolumn{1}{c}{ Fruiting response ${ }^{\prime}$} & Mean & s.D. \\
0 & $29,38,35,27,32,33$ & $32 \cdot 33$ & $3 \cdot 98$ \\
12 & $14,13,9,20,16,17$ & $13 \cdot 17$ & $4 \cdot 71$ \\
24 & $6,5,4,4,2,7$ & $4 \cdot 67$ & $1 \cdot 75$ \\
36 & $3,0,0,2,0,4$ & $1 \cdot 50$ & $1 \cdot 76$ \\
48 & $0,0,0,0,0,0$ & $0 \cdot 00$ & $0 \cdot 00$
\end{tabular}

* The period in darkness at the beginning of the 5 days following the placement of the FIS-containing agar cylinder.

$\dagger$ No. of stage II and III fruiting bodies/fruiting area of 6 replicate test plates at 8 days.

Table 5. Effect of different times of light treatment on the FIS-induced fruiting response

All cultures were in darkness for the 3 days preceding induction.

$\begin{array}{cccrc}\begin{array}{c}\text { Period of } \\ \begin{array}{c}\text { illumination } \\ \text { (h) }\end{array}\end{array} & \begin{array}{c}\text { Visible } \\ \text { differentiation } \\ \text { before dark } \\ \text { incubation }\end{array} & \text { Fruiting response† } & \text { Mean } & \text { S.D. } \\ 24 & \text { None } & \text { o, o, o, o, o, o } & 0.00 & 0.00 \\ 36 & \text { None } & 8,6,14,5,13,10 & 9 \cdot 88 & 3.13 \\ 48 & \text { Stage I } & 32,32,36,38,23,22 & 30 \cdot 50 & 6.62 \\ \text { I20 } & - & 29,38,35,27,32,33 & 32 \cdot 33 & 3.98\end{array}$

* Light period at the beginning of the 5 days following the placement of the FIS-containing agar cylinder.

$\dagger$ No. of stage II and III fruiting bodies/fruiting area of 6 replicate test plates at 8 days.

light for 5 days and scored for a fruiting reaction (Table 4). The longer the cultures had remained in the dark before exposure to light, the less the fruiting response. The fruiting structures that did form after 24 and $36 \mathrm{~h}$ darkness developed on the youngest hyphal area over the inducing cylinder.

Three-day-old dark-grown cultures were exposed to FIS and incubated in continuous light. After various times groups of six plates were placed in the dark, and at the end of the experiment, all cultures were scored for fruiting (Table 5). A minimum amount of light appeared to be necessary to elicit an FIS-induced fruiting response. The number of fruiting bodies, however, increased with the length of illumination and a maximum response was observed with the $48 \mathrm{~h}$ light regime. With regard to development, no differentiation was 
Table 6. Effect of high temperature on the FIS-induced fruiting response

All treatment was carried out under constant light of 60 to $80 \mathrm{ft}$-candle.

\begin{tabular}{|c|c|c|c|c|c|}
\hline $\begin{array}{c}\text { Temperature } \\
\text { before } \\
\text { placement } \\
\left({ }^{\circ} \mathrm{C}\right)\end{array}$ & $\begin{array}{l}\text { Temperature } \\
\text { after } \\
\text { placement } \\
\text { Ist day } \\
\left.\text { ( }{ }^{\circ} \mathrm{C}\right)\end{array}$ & $\begin{array}{l}\text { Incubation } \\
\text { period, } \\
4^{\text {th day }}\end{array}$ & Fruiting response $\dagger$ & Mean & S.D. \\
\hline 25 & 25 & 25 & $38,37,26,40,36,29$ & $34: 33$ & $5 \cdot 58$ \\
\hline 30 & 25 & 25 & $27,28,32,33,36,30$ & $3 I \cdot 00$ & $3 \cdot 35$ \\
\hline 25 & 30 & 30 & $0,0,0,0,0,0$ & 0.00 & 0.00 \\
\hline 25 & 25 & 25 & $0,0,0,0,0,0$ & 0.00 & 0.00 \\
\hline 25 & 30 & 25 & $21,34,35,10,15,28$ & $23 \cdot 83$ & $10 \cdot 22$ \\
\hline
\end{tabular}

detectable in cultures illuminated for $36 \mathrm{~h}$ before dark incubation. However, stage II and III fruiting structures developed in these cultures during the dark period.

These experiments showed that FIS did not replace the light requirement for fruiting, that the photoreaction could not precede exposure to FIS but had to follow it closely for an induced fruiting response to occur, and that fruiting body initiation and development could take place in the absence of light provided that illumination closely followed FIS exposure and was of sufficient duration.

\section{High temperature and FIS induction}

The effect of temperature on fruiting in $S$. commune has been studied by Niederpruem, Hobbs \& Henry (1964) and Perkins (1969), who found homokaryotic fruiting was inhibited at $30^{\circ} \mathrm{C}$. To determine when high temperature exerts its effect during fruiting body development, different sets of cultures were treated with different combinations of temperatures during three separate periods (Table 6).

High temperature treatment before induction did not have any effect on the induced fruiting response, whereas a temperature of $30^{\circ} \mathrm{C}$ during the 4 -day incubation period inhibited induced fruiting. Although high temperature during the first day of the induction period (when the mycelium was presumed to be taking up FIS) did not inhibit fruiting, it did result in considerable variability in the number of fruiting structures produced among the replicate cultures.

Thus temperature-sensitive reactions occurred during the incubation period following exposure to FIS. This period is characterized by the formation of fruiting hyphae which cohere and form the hyphal aggregates that eventually develop into fruiting bodies (Leonard, unpublished observations).

\section{DISCUSSION}

The present method for detecting FIS activity has a number of advantages over previous test systems. Because the induced fruiting reaction always occurs in a predictable, restricted area of the culture, it can be determined whether there are special regions for fruiting initiation. Growing the test colony on cellophane membrane allows manipulation of the inducing agar cylinder without disturbing the normal growth pattern of the hyphae, for the study of FIS absorption and other early induction processes. Quantities as low as 0.0I ml can be bioassayed, in contrast to the $\mathrm{I} \cdot 0$ to $2 \mathrm{ml}$ required previously (Leonard \& Dick, 
1968); this is attributed to the absence of unnecessary diffusion of FIS into the surrounding medium.

Leonard \& Dick (I968) described FIS-induced fruiting in Schizophyllum quantitatively as 'high', 'moderate', 'low', and 'none'. The quantitative relationship reported here between number of fruiting bodies per inducing cylinder and concentration of inducing extract, provides a method for quantitative assay. With sufficient replicates, the FIS activity of an unknown solution could be predicted from the number of fruiting bodies induced. For this purpose a parallel-line bioassay using a standard concentration of FIS could be employed (see Goldstein, 1964).

The failure of fruiting development to proceed beyond stage III into the expansion stages of IV and $\mathrm{V}$ with the present methods is not understood, but may be attributable to the relatively low nutrient level in the culture medium. It is an advantage, however, as large stage- $V$ fruit bodies would obstruct the entire fruiting area and further affect the variability of the fruiting reaction (Leonard \& Dick, I973). Since FIS acts as a triggering material, the expansion stages are of little consequence with regard to the bioassay and purification of substances responsible for FIS activity.

Only hyphal segments less than $24 \mathrm{~h}$ old could be induced to produce fruiting hyphae. Perkins (1969) observed photo-induction of dikaryotic fruiting bodies only in cells that were 2 to $26 \mathrm{~h}$ old during induction. That FIS induction could occur in colonies as young as one day suggested that no maturation process was required in FIS-induced homokaryotic fruiting, in contrast to a maturation requirement in dikaryotic fruiting (Perkins, 1969). Thus FIS-induced fruiting may be a localized event which does not alter the metabolic pathway of the whole colony. However, the whole colony could affect fruiting development in localized FIS-induced areas, as demonstrated by the increased variability of the FISinduced fruiting response in older colonies.

There are conflicting reports concerning the light requirement for fruiting in S. commune (Jürgens, 1958; Raper \& Krongelb, 1958; Niederpruem et al. 1964; Perkins, 1969). In dikaryotic fruiting, light was required for the formation of stage III fruiting structures; stages I and II could form in the absence of light. Nevertheless, early exposure to light results in more abundant stage II structures and also speeds up the rate of fruiting development (Perkins, 1969). On the other hand, complete dikaryotic fruiting responses occur in the dark when the colonies are cultured in a rich nitrogen-containing medium, or in the presence of KOH vapours (Perkins, r969).

In FIS-induced homokaryotic fruiting, a light period must closely follow the FIS stimulus for fruiting morphogenesis to occur. Light appears to play a fundamental role with FIS in stimulating the complete potential for differentiation: fruiting bodies appeared on FISexposed mycelia that had been grown in the light for $36 \mathrm{~h}$ and then placed in the dark before any differentiation was visible (Table 5), whereas FIS-exposed cultures given insufficient or no light failed to differentiate fruiting structures.

Although it is not known what metabolic events occur after exposure to FIS and light, temperature studies indicated that important heat-labile reactions occur (Table 6). Whether the heat-labile steps are involved with the initiation of a new kind of hypha, the fruiting hypha, or the development of hyphal aggregates, or even earlier steps in fruiting morphogenesis, awaits elucidation.

Although many of the studies were carried out with the hope of defining optimal conditions for the assay of fruiting morphogens, it is clear that experiments along these lines also promise to further our understanding of the fungal colony and its substructure. 
The generosity of the Kentucky Mushroom Farm, Lawton, Kentucky, U.S.A., in providing Agaricus bisporus fruiting bodies is gratefully acknowledged.

\section{REFERENCES}

BeVAN, E. A. \& Kemp, R. F. O. (1958). Stipe regeneration and fruit-body production in Collybia velutipes (Curt.) Fr. Nature, London 181, I 145-1 146.

BURNETT, J. H. (1968). Fundamentals of Mycology, p. 76. New York: St Martin's Press.

EGER, G. (1965). Untersuchungen über die Bildung und Regeneration von Fruchtkörpern bei Hutpilzen. I. Pleurotus florida. Archiv fuir Mikrobiologie 50, 343-356.

GoLDSTEIN, A. (1964). Biostatistics, an Introductory Text. New York: Macmillan.

JürgENS, C. (1958). Physiologische and Genetische Untersuchungen über die Fruchtkörperbildung bei Schizophyllum commune. Archiv für Mikrobiologie 31, 388-42 I.

LEONARD, T. J. \& Dick, S. (1968). Chemical induction of haploid fruiting in Schizophyllum commune. Proceedings of the National Academy of Sciences of the United States of America 59, 745-751.

LEONARD, T. J. \& DiCK, S. (1973). Induction of haploid fruiting by mechanical injury in Schizophyllum commune. Mycologia 65, 809-822.

Niedekpruem, D. J., HoBBs, H. \& HeNRY, L. (1964). Nutritional studies of development in Schizophyllum commune. Journal of Bacteriology 88, I721-1729.

Park, D. \& Robinson, P. M. (1966). Aspects of hyphal morphogenesis. In Trends in Plant Morphogenesis, pp. 27-66. Edited by E. Cutter. London: Longmans.

Perkins, J. H. (1969). Morphogenesis in Schizophyllum commune. I. Effects of white light. Plant Physiology 44, 1706-171I.

RAPER, J. R. (1966). Genetics of Sexuality in Higher Fungi. New York: Ronald Press.

RAPER, J. R. \& KRONGELB, G. S. (1958). Genetic and environmental aspects of fruiting in Schizophyllum commune. Mycologia 50, 707-740.

SoKal, R. R. \& ROHLF, F. J. (1969). Biometry. San Francisco: Freeman.

TABER, W. A. (1966). Morphogenesis in Basidiomycetes. In The Fungi, vol. 2, pp. 387-412. Edited by C. C. Ainsworth and A. S. Sussman. New York and London: Academic Press.

UNo, I. \& IsHIKAwA, T. (1973). Purification and identification of fruiting inducing substances in Coprinus macrorhizus. Journal of Bacteriology 113, 1240-1248.

URAYAMA, T. (1956). Preliminary note on the stimulative effect of certain specific bacteria upon fruit body formation in Psilocybe panaeoliformis Murrill. Botanical Magazine (Tokyo) 70, 29-30. 